\title{
Statistical Limit Theorems for Suspension Flows
}

\author{
Ian Melbourne \\ Department of Maths and Stats \\ University of Surrey \\ Guildford GU2 7XH, UK
}

\author{
Andrei Török * \\ Department of Mathematics \\ University of Houston \\ Houston, TX 77204-3008, USA
}

May 3, 2004

\begin{abstract}
In dynamical systems theory, a standard method for passing from discrete time to continuous time is to construct the suspension flow under a roof function. In this paper, we give conditions under which statistical laws, such as the central limit theorem and almost sure invariance principle, for the underlying discrete time system are inherited by the suspension flow.

As a consequence, we give a simpler proof of the results of Ratner (1973) and recover the results of Denker and Philipp (1984) for Axiom A flows. Moreover, we obtain several new results for nonuniformly and partially hyperbolic flows, including frame flows on negatively curved manifolds satisfying a pinching condition.
\end{abstract}

\section{Introduction}

Let $(X, \mu)$ be a probability space. Suppose that $S: X \rightarrow X$ is a measure-preserving transformation and that $\Phi: X \rightarrow \mathbb{R}$ is a measurable observation. Consider the sequence of partial sums $\Phi_{N}=\sum_{j=0}^{N-1} \Phi \circ S^{j}$. Provided $S$ is ergodic and $\Phi \in L^{1}(X)$, Birkhoff's ergodic theorem (or the strong law of large numbers) states that

(SLLN) $\quad \lim _{N \rightarrow \infty} \frac{\Phi_{N}}{N}=\int_{X} \Phi d \mu$ a.e.

Under certain hypotheses, the central limit theorem (CLT) states that

\footnotetext{
*and Institute of Mathematics of the Romanian Academy, P.O. Box 1-764, RO-70700 Bucharest, Romania
} 
(CLT) $\quad \lim _{N \rightarrow \infty} \mu\left\{\frac{\Phi_{N}-N \int_{X} \Phi d \mu}{\sqrt{N}}<c\right\}=\frac{1}{\sqrt{2 \pi \sigma^{2}}} \int_{-\infty}^{c} e^{-x^{2} / 2 \sigma^{2}} d x$.

The CLT has been established for large classes of dynamical systems. Usually the dynamical system $X$ is assumed to have some hyperbolicity properties (Axiom A or nonuniformly/partially hyperbolic, see for example $[25,27,30,31,7,11,22]$ ) or expansivity properties (for example $[28,19]$ ), and the observation $\Phi$ is assumed to have certain regularity properties such as Hölder continuity or bounded variation.

Suspension flows There is a standard method for constructing continuous time dynamical systems (or flows) from a discrete time dynamical system $S: X \rightarrow X$.

A measurable function $r: X \rightarrow \mathbb{R}$ is called a roof function if $r$ is positive almost everywhere and $r \in L^{1}$. Define the suspension

$$
X_{r}=\{(x, u) \in X \times \mathbb{R}: 0 \leq u \leq r(x)\} / \sim,
$$

where $(x, r(x))$ is identified with $(S x, 0)$. The suspension flow $S_{t}: X_{r} \rightarrow X_{r}$ is given by $S_{t}(x, u)=(x, u+t)$ computed subject to the identifications. (If $S: X \rightarrow X$ is not invertible, then $S_{t}$ is a semi-flow, but we shall still speak of suspension flows.)

Let $\bar{r}=\int_{X} r d \mu$. Then $\mu_{r}=\mu \times \ell / \bar{r}$ is an invariant probability measure for the suspension flow, where $\ell$ stands for the Lebesgue measure on the real line. Given an $L^{2}$ observation $\phi: X_{r} \rightarrow \mathbb{R}$, we consider the family of integrals $\phi_{T}=\int_{0}^{T} \phi \circ S_{t} d t$.

Central limit theorems for suspension flows (convergence in distribution of $\left.\frac{1}{\sqrt{T}}\left(\phi_{T}-T \int_{X_{r}} \phi d \mu_{r}\right)\right)$ were obtained by Ratner [25] for Hölder observations in the Anosov context. It follows from Bowen [3] that Ratner's results hold for general Axiom A flows. Denker and Philipp [9] gave a relatively elementary proof of Ratner's theorem, using the fact that the hyperbolic diffeomorphism $S: X \rightarrow X$ satisfies strong statistical properties, in particular the almost sure invariance principle (ASIP).

In this paper, we give mild conditions under which the CLT for a general suspension flow $S_{t}: X_{r} \rightarrow X_{r}$ follows from the corresponding result for the discrete dynamical system $S: X \rightarrow X$. Our proof is not constrained to the hyperbolic context and is more general, and simpler, than the original proof of [25]. In addition our proof does not rely on the ASIP. A major advantage of our approach is that the roof function $r$ need not be bounded. Hence, we are able to prove the CLT in a number of situations where $[9,25]$ do not apply, see Section 5.

We assume throughout that $\phi: X_{r} \rightarrow \mathbb{R}$ is an observation of mean zero, and $r \in L^{a}(X), \phi \in L^{b}\left(X_{r}\right)$, where $a, b$ are moderately large. We define

$$
\Phi(x)=\int_{0}^{r(x)} \phi(x, u) d u .
$$

Then $\Phi: X \rightarrow \mathbb{R}$ is a mean zero observation on $X$, and the aim is to deduce statistical properties of $\phi$ on the suspension flow from the corresponding statistical properties of $\Phi$ on the base dynamics. 
Central Limit Theorem The first version of our main theorem is as follows. In this result, we do not require invertibility of $S: X \rightarrow X$.

Theorem 1.1 Let $S: X \rightarrow X$ be an ergodic transformation. Suppose that 1 $\left.\frac{1}{a}\right)\left(1-\frac{1}{b}\right) \geq \frac{1}{2}$. Suppose that $\Phi$ and $r$ each satisfy the CLT. Then $\phi$ satisfies the CLT. Moreover, if the CLT for $\Phi$ has variance $\sigma_{1}^{2} \geq 0$, then the CLT for $\phi$ has variance $\sigma^{2}=\sigma_{1}^{2} / \bar{r}$.

Remark 1.2 (a) The regularity hypothesis $\left(1-\frac{1}{a}\right)\left(1-\frac{1}{b}\right) \geq \frac{1}{2}$ is optimal in the sense that otherwise $\Phi$ need not lie in $L^{2}$ (see Remark 2.4).

(b) The proof of Ratner [25] relies heavily on the additional assumptions that $(\Phi, r)$ jointly satisfy a 2-dimensional CLT, and that $\Phi \in L^{\infty}$.

(c) We do not require that the CLTs for $\Phi$ and $r$ are nondegenerate (they may have zero variance). Moreover, the result remains true if $\frac{1}{\sqrt{N}}\left(r_{N}-N \bar{r}\right)$ converges in distribution, regardless of the limit distribution.

We have the following generalization of Theorem 1.1.

Theorem 1.3 Let $S: X \rightarrow X$ be an invertible ergodic transformation. Suppose that $\left(1-\frac{1}{a}\right)\left(1-\frac{1}{b}\right) \geq \frac{1}{2}$ and that $\frac{1}{\sqrt{N}} \Phi_{N} \rightarrow_{d} G$. Suppose further that there exist $\alpha \in(0,1]$, $\beta \in(0,1)$ with $\alpha \beta \leq \frac{1}{2}$ such that

(i) $\frac{1}{|N|^{\alpha}} \Phi_{N} \rightarrow 0$ a.e. as $N \rightarrow \pm \infty$ (where $\Phi_{N}=-\sum_{j=1}^{|N|} \Phi \circ S^{-j}$ for $N<0$ ), and

(ii) $\frac{1}{N^{\beta}}\left(r_{N}-N \bar{r}\right)$ converges in distribution as $N \rightarrow \infty$.

Then $\frac{1}{\sqrt{T}} \phi_{T} \rightarrow{ }_{d} G / \sqrt{\bar{r}}$ as $T \rightarrow \infty$.

Remark 1.4 (a) For invertible dynamical systems, the SLLN and the CLT hold in backwards time $(N, T \rightarrow-\infty)$ if and only if they hold in forwards time. Hence in Theorem 1.1 it is not necessary to assume that $S: X \rightarrow X$ is invertible, since we can pass to the natural extension. In particular, Theorem 1.1 is a special case of Theorem 1.3 with $\alpha=1$ and $\beta=\frac{1}{2}$. Theorem 1.3 shows that the CLT condition on $r$ can be weakened when the SLLN condition on $\Phi$ is strengthened.

(b) In many situations, including the hyperbolic setting, hypotheses (i) and (ii) of Theorem 1.3 are valid for all $\alpha, \beta>\frac{1}{2}$, so certainly $\alpha \beta \leq \frac{1}{2}$.

(c) For ease of exposition, we have focused on convergence in distribution for which the normalizing factors are $\sqrt{N}$ and $\sqrt{T}$. It is easy to generalize Theorem 1.3 to allow for different normalizing factors. Let $c>0$. Assume that $a, b \geq 1$ satisfy $\left(1-\frac{1}{a}\right)\left(1-\frac{1}{b}\right) \geq\left(1-\frac{1}{c}\right)$ and that $\alpha \in(0,1], \beta \in(0,1)$ satisfy $\alpha \beta \leq c$. Let $r \in L^{a}(X)$, $\Phi \in L^{b}\left(X_{r}\right)$ satisfy conditions (i) and (ii) of Theorem 1.3 for the revised values of $\alpha$ and $\beta$. If $\frac{1}{N^{c}} \Phi_{N} \rightarrow{ }_{d} G$, then $\frac{1}{T^{c}} \phi_{T} \rightarrow{ }_{d} G / \bar{r}^{c}$. 
Conjecture 1.5 The conclusion of Theorem 1.3 remains valid if, instead of conditions (i) and (ii), there exists $\beta \in(0,1)$ such that

$$
\frac{1}{|N|^{\beta}}\left(r_{N}-N \bar{r}\right) \rightarrow 0 \text { a.e. as } N \rightarrow \pm \infty
$$

Other limit laws Our results on the CLT extend in a straightforward way to the functional central limit theorem (or weak invariance principle). For example, suppose that $S: X \rightarrow X$ is ergodic, and let $r \in L^{\infty}(X)$ and $\phi \in L^{2}\left(X_{r}\right)$. Suppose that $r$ satisfies the CLT. If $\Phi$ satisfies the functional CLT, then so does $\phi$.

We also consider almost sure results such as the law of the iterated logarithm (LIL) and the ASIP, generalising and simplifying results of Wong [29] and Denker and Philipp [9] respectively. The arguments are easier than for the CLT.

We note that the corresponding results for time-one maps of hyperbolic flows are more delicate than those for the flow itself. See [11, 22].

The remainder of the paper is organized as follows. Section 2 contains an approximation result. Our main results on the CLT are proved in Section 3. In Section 4, we prove the LIL and ASIP for suspension flows. In Section 5, we apply our results to nonuniformly hyperbolic and partially hyperbolic flows, including frame flows on negatively curved manifolds satisfying a pinching condition.

\section{An approximation result}

Throughout this section, we assume that $r: X \rightarrow \mathbb{R}$ is a roof function, so $r$ is positive almost everywhere, and $r \in L^{1}(X)$ with mean $\bar{r}=\int_{X} r d \mu$. We assume that $S: X \rightarrow X$ is measure-preserving and that $r$ satisfies the SLLN, so

$$
r_{N}=N \bar{r}+o(N) \text { as } N \rightarrow \infty
$$

almost everywhere, where $r_{N}(x)=r(x)+r(S x)+\cdots+r\left(S^{N-1} x\right)$.

It follows from (2.1) that $\lim _{N \rightarrow \infty} r_{N}=\infty$ almost everywhere. Given $T>0$, we define $n[x, T]$ to be the largest integer $n$ such that $r_{n}(x) \leq T$. That is

$$
r_{n[x, T]}(x) \leq T<r_{n[x, T]+1}(x) .
$$

For almost every $x \in X, n[x, T]$ is defined for all $T>0$ and $\lim _{T \rightarrow \infty} n[x, T]=\infty$ a.e. By the ergodic theorem, it follows easily (cf. [8, Lemma 11.2.1]) that

$$
\lim _{T \rightarrow \infty} \frac{T}{n[x, T]}=\bar{r} \text { a.e. }
$$

Given $\phi: X_{r} \rightarrow \mathbb{R}$, we define $\Phi(x)=\int_{0}^{r(x)} \phi(x, u) d u$ as usual, and $\Psi(x)=$ $\int_{0}^{r(x)}|\phi(x, u)| d u$. We have the following basic approximation result: 
Lemma 2.1 Suppose that $1 \leq a, b, p \leq \infty$, and $\left(1-\frac{1}{a}\right)\left(1-\frac{1}{b}\right) \geq\left(1-\frac{1}{p}\right)$. Let $r \in L^{a}(X), \phi \in L^{b}\left(X_{r}\right)$. Then

(a) $\phi_{T}(x, 0)=\Phi_{n[x, T]}(x)+o\left(T^{1 / p}\right)$ as $T \rightarrow \infty$, for almost all $x \in X$, and

(b) $\frac{1}{T^{1 / p}}\left(\phi_{T}(x, u)-\Phi_{n[x, T]}(x)\right) \rightarrow 0$ as $T \rightarrow \infty$, in probability (on $\left.X_{r}\right)$.

Remark 2.2 If $a=b=\infty$ in Lemma 2.1, then $\phi_{T}(x, u), \phi_{T}(x, 0)$ and $\Phi_{n[x, T]}(x)$ differ by amounts that are $O(1)$ almost everywhere.

The remainder of this section is concerned with the proof of Lemma 2.1.

Proposition 2.3 Suppose that $1 \leq a, b, p \leq \infty$, and $\left(1-\frac{1}{a}\right)\left(1-\frac{1}{b}\right) \geq\left(1-\frac{1}{p}\right)$. Let $r \in L^{a}(X), \phi \in L^{b}\left(X_{r}\right)$. Then $\Phi, \Psi \in L^{p}(X)$ and $\|\Phi\|_{p} \leq\|\Psi\|_{p} \leq\|r\|_{a}\|\phi\|_{b}$.

Proof Assume $1<a, b, p<\infty$, hence $b<p$ (the other cases are easier).

By Hölder's inequality with $1 / b^{\prime}+1 / b=1$, we obtain

$$
\Psi(x) \leq\left(\int_{0}^{r(x)} 1 d t\right)^{1 / b^{\prime}}\left(\int_{0}^{r(x)}|\phi(x, t)|^{b} d t\right)^{1 / b}=r(x)^{(b-1) / b}\left(\int_{0}^{r(x)}|\phi(x, t)|^{b} d t\right)^{1 / b} .
$$

We apply Hölder's inequality once more with $1 / c+1 / d=1$ where $d=b / p$, hence $c=b /(b-p)$, to obtain

$$
\begin{aligned}
\int_{X} \Psi(x)^{p} d \mu & \leq \int_{X} r(x)^{p(b-1) / b}\left(\int_{0}^{r(x)}|\phi(x, t)|^{b} d t\right)^{p / b} d \mu \\
& =\int_{X} r(x)^{p(b-1) / b}\left(\int_{0}^{r(x)}|\phi(x, t)|^{b} d t\right)^{1 / d} d \mu \\
& \leq\left(\int_{X} r(x)^{c p(b-1) / b} d \mu\right)^{1 / c}\left(\int_{X} \int_{0}^{r(x)}|\phi(x, t)|^{b} d t d \mu\right)^{1 / d}
\end{aligned}
$$

which is finite provided that $r \in L^{p(b-1) /(b-p)}(X)$ and $\phi \in L^{b}\left(X_{r}\right)$.

Setting $p(b-1) /(b-p)=a$ leads to the required condition.

Remark 2.4 The value of $p$ is optimal, as can be seen from the following examples. If $a<\infty$, given $r \in L^{a}(X)$, take $\phi(x, t)=r(x)^{(a-1) / b}$; then $\phi \in L^{b}\left(X_{r}\right)$ and $\|\Phi\|_{p}=$ $\|\Psi\|_{p}=\|r\|_{a}^{a / p}$, where $\left(1-\frac{1}{a}\right)\left(1-\frac{1}{b}\right)=\left(1-\frac{1}{p}\right)$. If $a=\infty$ take $\phi(x, t)=c(x)$ with $c \in L^{b}(X)$ and $\operatorname{supp}(c) \subset\left\{r \geq\|r\|_{\infty} / 2\right\}$.

For fixed $N \geq 1$, it is easily proved by induction that

$$
\phi_{r_{N}(x)}(x, 0)=\Phi_{N}(x) .
$$

Hence $\left|\phi_{T}(x, 0)-\Phi_{n[x, T]}(x)\right| \leq A_{T}(x)$, where $A_{T}(x)=\int_{r_{n[x, T]}(x)}^{T}\left|\phi \circ S_{t}(x, 0)\right| d t$. 
Proposition 2.5 Suppose that $r$ satisfies the $S L L N$ (2.1) and $\Psi \in L^{p}(X)$ for some $p \geq 1$. Then $A_{T}=o\left(T^{1 / p}\right)$ as $T \rightarrow \infty$ a.e.

Proof For $N=n[x, T]$ compute that

$$
\begin{aligned}
A_{T}(x) & \leq \int_{r_{N}(x)}^{r_{N+1}(x)}\left|\phi \circ S_{t}(x, 0)\right| d t=\int_{0}^{r\left(S^{N} x\right)}\left|\phi \circ S_{t} \circ S_{r_{N}(x)}(x, 0)\right| d t \\
& =\int_{0}^{r\left(S^{N} x\right)}\left|\phi \circ S_{t}\left(S^{N} x, 0\right)\right| d t=\Psi\left(S^{N} x\right) .
\end{aligned}
$$

Since $\Psi \in L^{p}(X)$, the ergodic theorem applies to $\Psi^{p} \in L^{1}(X)$ so that $\Psi^{p}\left(S^{n} x\right)=o(n)$ almost everywhere. Hence, $\Psi\left(S^{n} x\right)=o\left(n^{1 / p}\right)$ almost everywhere. It follows that $A_{T}=$ $o\left(n[x, T]^{1 / p}\right)$ almost everywhere. By $(2.3), A_{T}(x)=o\left(T^{1 / p}\right)$ almost everywhere.

Part (a) of Lemma 2.1 follows immediately from Propositions 2.3 and 2.5. To prove part (b), compute that

$$
\phi_{T}(x, u)=\int_{0}^{T} \phi \circ S_{t}(x, u) d t=\int_{0}^{T} \phi \circ S_{t+u}(x, 0) d t=\int_{u}^{T+u} \phi \circ S_{t}(x, 0) d t
$$

and so by (2.4),

$$
\begin{aligned}
\left|\phi_{T}(x, u)-\Phi_{n[x, T]}(x)\right| & =\left|\phi_{T}(x, u)-\phi_{r_{n[x, T]}(x)}(x, 0)\right| \\
& =\left|\int_{u}^{T+u} \phi \circ S_{t}(x, 0) d t-\int_{0}^{r_{n[x, T]}(x)} \phi \circ S_{t}(x, 0) d t\right| \\
& \leq A_{T}(x)+B_{T}(x, u),
\end{aligned}
$$

where $B_{T}(x, u)=\int_{T}^{T+u}\left|\phi \circ S_{t}(x, 0)\right| d t+\int_{0}^{u}\left|\phi \circ S_{t}(x, 0)\right| d t$ for $u \in[0, r(x)]$.

Proposition 2.6 If $r \in L^{1}(X), \phi \in L^{1}\left(X_{r}\right)$, and $q>0$, then $\frac{1}{T^{q}} B_{T}$ converges to zero in probability.

Proof It suffices to estimate the first term in the definition of $B_{T}$, so we write $B_{T}(x, u)=\int_{T}^{T+u}\left|\phi \circ S_{t}(x, 0)\right| d t$. Define $V_{T}(x)=B_{T}(x, r(x))=\int_{0}^{r(x)}\left|\phi \circ S_{T}(x, t)\right| d t$. Since $S_{T}$ is measure-preserving, $\left\|V_{T}\right\|_{1}=\bar{r}\|\phi\|_{1}$ and so $V_{T} / T^{q} \rightarrow 0$ in $L^{1}$. In particular, $V_{T} / T^{q} \rightarrow 0$ in probability. Fix $\varepsilon>0$ and define

$$
E_{T}=\left\{x \in X: V_{T}(x) / T^{q} \geq \varepsilon\right\}, \quad F_{T}=\left\{(x, u) \in X_{r}: B_{T}(x, u) / T^{q} \geq \varepsilon\right\} .
$$

Then $\mu\left(E_{T}\right) \rightarrow 0$. Since $B_{T}(x, u) \leq V_{T}(x)$ and $r \in L^{1}$, it follows that $\mu_{r}\left(F_{T}\right) \leq$ $(1 / \bar{r}) \int_{E_{T}} r d \mu \rightarrow 0$. Hence $B_{T} / T^{q} \rightarrow 0$ in probability as required.

Part (b) of Lemma 2.1 is immediate from equation (2.5) and Propositions 2.3, 2.5 and 2.6 (with $q=1 / p$.) 


\section{Central Limit Theorem}

In this section, we prove Theorem 1.3 extending the central limit theorem (CLT) from observations on $X$ to observations on the suspension flow $X_{r}$.

Since two different measures $\mu$ and $\mu_{r}$ are involved, the following device for passing between $X$ and $X_{r}$ is required.

Lemma 3.1 Let $S: X \rightarrow X$ be ergodic and let $X_{r}$ be the suspension with respect to the $L^{1}$ roof function $r$. Let $\Phi: X \rightarrow \mathbb{R}$ be an $L^{2}$ observation and set $\Phi_{N}=$ $\sum_{j=0}^{N-1} \Phi \circ S^{j}$.

Define $\widehat{\Phi}_{N}: X_{r} \rightarrow \mathbb{R}$ by $\widehat{\Phi}_{N}(x, u)=\Phi_{N}(x)$. If $\Phi_{N} / \sqrt{N} \rightarrow{ }_{d} G$ on $X$, then $\widehat{\Phi}_{N} / \sqrt{N} \rightarrow_{d} G$ on $X_{r}$.

Proof It follows from Eagleson [12, Section 4.2a] (see also [1, Section 3.6]) that $\Phi_{N} / \sqrt{N}$ converges in distribution to $G$ relative to any probability measure $\nu$ that is absolutely continuous with respect to the underlying ergodic measure $\mu$. Taking $\nu$ to be the measure with density $r / \bar{r}$, we have $\int_{X} e^{i t \Phi_{N} / \sqrt{N}} r / \bar{r} d \mu \rightarrow E\left(e^{i t G}\right)$ for all $t \in \mathbb{R}$. But

$$
\int_{X_{r}} e^{i t \widehat{\Phi}_{N} / \sqrt{N}} d \mu_{r}=(1 / \bar{r}) \int_{X} \int_{0}^{r} e^{i t \Phi_{N} / \sqrt{N}} d(\mu \times \ell)=\int_{X} e^{i t \Phi_{N} / \sqrt{N}} r / \bar{r} d \mu .
$$

Hence $\int_{X_{r}} e^{i t \widehat{\Phi}_{N} / \sqrt{N}} d \mu_{r} \rightarrow E\left(e^{i t G}\right)$ and so $\widehat{\Phi}_{N} / \sqrt{N} \rightarrow{ }_{d} G$.

Remark 3.2 The result of Eagleson [12] used in the above argument states that central limit theorems for ergodic sequences are automatically "mixing in the sense of Rényi" (a concept introduced in [26]).

Now let $\phi: X_{r} \rightarrow \mathbb{R}$ be a mean zero observation. We assume that $r \in L^{a}(X)$, $\phi \in L^{b}\left(X_{r}\right)$ where $\left(1-\frac{1}{a}\right)\left(1-\frac{1}{b}\right) \geq \frac{1}{2}$. Recall that $\Phi(x)=\int_{0}^{r(x)} \phi(x, u) d u$ and define $\widehat{\Phi}(x, u)=\Phi(x)$ as in Lemma 3.1. Let $S: X \rightarrow X$ and $\Phi_{N}, N \in \mathbb{Z}$, be as described in Theorem 1.3. Define

$$
m[x, T]=n[x, T]-[T / \bar{r}] \quad \text { and } \quad W_{T}(x)=\frac{1}{\sqrt{T}} \Phi_{m[x, T]}\left(S^{[T / \bar{r}]} x\right) .
$$

Lemma 3.3 Suppose that $r \in L^{1}(X)$ satisfies the $\operatorname{SLLN}(2.1)$ and that

$$
W_{T} \rightarrow{ }_{d} 0 \text { on } X \quad \text { and } \frac{1}{\sqrt{N}} \widehat{\Phi}_{N} \rightarrow{ }_{d} G \text { on } X_{r} \text { as } T, N \rightarrow \infty \text {. }
$$

Then

$$
\frac{1}{\sqrt{T}} \widehat{\Phi}_{n[x, T]} \rightarrow{ }_{d} G / \sqrt{\bar{r}} \text { on } X_{r}
$$


Proof By (2.3), it is equivalent to show that $\frac{1}{\sqrt{n[x, T]}} \widehat{\Phi}_{n[x, T]}(x, u) \rightarrow_{d} G$ on $X_{r}$. We compute that

$$
\begin{aligned}
\frac{1}{\sqrt{n[x, T]}} \widehat{\Phi}_{n[x, T]}(x, u)-\frac{1}{\sqrt{[T / \bar{r}]}} \widehat{\Phi}_{[T / \bar{r}]}(x, u)= & \frac{1}{\sqrt{n[x, T]}}\left(\widehat{\Phi}_{n[x, T]}(x, u)-\widehat{\Phi}_{[T / \bar{r}]}(x, u)\right) \\
& +\left(\frac{1}{\sqrt{n[x, T]}}-\frac{1}{\sqrt{[T / \bar{r}]}}\right) \widehat{\Phi}_{[T / \bar{r}]}(x, u) \\
& =\mathrm{I}+\mathrm{II} .
\end{aligned}
$$

By definition of $\widehat{\Phi}_{N}$, term I is independent of $u$ and

$$
\begin{aligned}
\mathrm{I} & =\frac{1}{\sqrt{n[x, T]}}\left(\Phi_{[T / \bar{r}]+m[x, T]}(x)-\Phi_{[T / \bar{r}]}(x)\right)=\frac{\sqrt{T}}{\sqrt{n[x, T]}} \frac{1}{\sqrt{T}} \Phi_{m[x, T]}\left(S^{[T / \bar{r}]} x\right) \\
& =\frac{\sqrt{T}}{\sqrt{n[x, T]}} W_{T}(x) \rightarrow{ }_{d} 0 \text { on } X
\end{aligned}
$$

by (2.3) and the hypothesis on $W_{T}$. Since $r \in L^{1}(X)$, it follows that $\mathrm{I} \rightarrow{ }_{d} 0$ on $X_{r}$.

Next, working on $X_{r}$,

$$
\mathrm{II}=\left(\frac{\sqrt{[T / \bar{r}]}}{\sqrt{n[x, T]}}-1\right)\left(\frac{1}{\sqrt{[T / \bar{r}]}} \widehat{\Phi}_{[T / \bar{r}]}(x, u)\right) .
$$

The second factor converges in distribution. The first factor converges (by (2.3)) to 0 a.e. Hence II $\rightarrow_{d} 0$ on $X_{r}$.

The key part of the argument is to verify that $W_{T} \rightarrow_{d} 0$. This can be carried out on $X$, with no mention of the suspension $X_{r}$.

Lemma 3.4 Let $r, \Phi \in L^{1}(X), r>0$. Suppose that there exist $\alpha \in(0,1], \beta \in(0,1)$ with $\alpha \beta \leq \frac{1}{2}$ such that

(i) $\frac{1}{|N|^{\alpha}} \Phi_{N} \rightarrow 0$ a.e. as $N \rightarrow \pm \infty$, and

(ii) $\frac{1}{N^{\beta}}\left(r_{N}-N \bar{r}\right)$ converges in distribution as $N \rightarrow \infty$.

Then $W_{T} \rightarrow{ }_{d} 0$ as $T \rightarrow \infty$.

Proof Fix $a>0$. We show that $\mu\left(\left|W_{T}\right| \geq a\right) \rightarrow 0$ as $T \rightarrow \infty$.

Let $\varepsilon>0$. By hypothesis (i), we can choose a set $\widetilde{X} \subset X$ with $\mu(\tilde{X})>1-\varepsilon$ and $N_{0}$ such that $\left|\frac{1}{M^{\alpha}} \Phi_{M}\right|<\varepsilon$ on $\widetilde{X}$ for all $|M| \geq N_{0}$. For each value of $T$, we define

$$
X_{T}^{\prime}=\left\{x \in X:|m[x, T]| \leq N_{0}\right\}, \quad X_{T}^{\prime \prime}=\left\{x \in X:|m[x, T]|>N_{0}\right\} .
$$


First, we estimate the contribution from $X_{T}^{\prime}$. For $x \in X_{T}^{\prime}$, we have

$$
\left|W_{T}(x)\right| \leq \frac{1}{\sqrt{T}} \sum_{j=-N_{0}}^{N_{0}-1}\left|\Phi \circ S^{j}\left(S^{[T / \bar{r}]}(x)\right)\right|=\frac{1}{\sqrt{T}} \Psi\left(S^{[T / \bar{r}]}(x)\right),
$$

where $\Psi=\sum_{j=-N_{0}}^{N_{0}-1}\left|\Phi \circ S^{j}\right|$. Since $\Psi$ lies in $L^{1}$, there is a constant $C$ and a subset $Y \subset$ $X$ with $\mu(Y)>1-\varepsilon$ such that $\Psi(y) \leq C$ for all $y \in Y$. Let $Y_{T}=X_{T}^{\prime} \cap\left(S^{[T / \bar{r}]}\right)^{-1}(Y)$. Then $\mu\left(Y_{T}\right)>\mu\left(X_{T}^{\prime}\right)-\varepsilon$ (since $S$ is measure-preserving), and $\Psi\left(S^{[T / \bar{r}]}(x)\right)<C$ for all $x \in Y_{T}$. Hence

$$
\begin{aligned}
\mu\left(x \in X_{T}^{\prime}:\left|W_{T}(x)\right| \geq a\right) & <\mu\left(x \in Y_{T}:\left|W_{T}(x)\right| \geq a\right)+\varepsilon \\
& \leq \mu\left(x \in Y_{T}: \frac{1}{\sqrt{T}} C \geq a\right)+\varepsilon=\varepsilon,
\end{aligned}
$$

for $T$ sufficiently large. Therefore, we can choose $T_{0}$ so that

$$
\mu\left(\left|W_{T}\right| \geq a\right) \leq \mu\left(x \in X_{T}^{\prime \prime}:\left|W_{T}(x)\right| \geq a\right)+\varepsilon, \text { for all } T \geq T_{0} .
$$

Next, we estimate the contribution from $X_{T}^{\prime \prime}$. Let $\tilde{X}_{T}^{\prime \prime}=X_{T}^{\prime \prime} \cap\left(S^{[T / \bar{r}]}\right)^{-1}(\widetilde{X})$ and note that $\mu\left(\tilde{X}_{T}^{\prime \prime}\right) \geq \mu\left(X_{T}^{\prime \prime}\right)-\varepsilon$ (since $S$ is measure-preserving). Hence,

$$
\mu\left(\left|W_{T}\right| \geq a\right) \leq \mu\left(x \in \tilde{X}_{T}^{\prime \prime}:\left|W_{T}(x)\right| \geq a\right)+2 \varepsilon .
$$

Write

$$
W_{T}(x)=\frac{|m[x, T]|^{\alpha}}{\sqrt{T}} \frac{1}{|m[x, T]|^{\alpha}} \Phi_{m[x, T]}\left(S^{[T / \bar{r}]}(x)\right) .
$$

On $X_{T}^{\prime \prime}$, we have $|m[x, T]|>N_{0}$ and so $\left|\frac{1}{|m[x, T]|^{\alpha}} \Phi_{m[x, T]}(y)\right|<\varepsilon$ for all $x \in X_{T}^{\prime \prime}$ and $y \in \widetilde{X}$. It follows from the definitions that $\left|\frac{1}{|m[x, T]|^{\alpha}} \Phi_{m[x, T]}\left(S^{[T / \bar{r}]}(x)\right)\right|<\varepsilon$ on $\widetilde{X}_{T}^{\prime \prime}$. Hence $\left|W_{T}(x)\right|<\varepsilon \frac{|m[x, T]|^{\alpha}}{\sqrt{T}}$ for $x \in \tilde{X}_{T}^{\prime \prime}$, so that

$$
\begin{aligned}
\mu\left(\left|W_{T}\right| \geq a\right) & \leq \mu\left(x \in \tilde{X}_{T}^{\prime \prime}: \frac{|m[x, T]|^{\alpha}}{\sqrt{T}} \geq a / \varepsilon\right)+2 \varepsilon \\
& \leq \mu\left(\frac{|m[x, T]|}{T^{1 /(2 \alpha)}} \geq(a / \varepsilon)^{1 / \alpha}\right)+2 \varepsilon \leq \mu\left(\frac{|m[x, T]|}{T^{\beta}} \geq(a / \varepsilon)^{1 / \alpha}\right)+2 \varepsilon
\end{aligned}
$$

since $\alpha \beta \leq \frac{1}{2}$. A direct calculation starting from hypothesis (ii) shows that the sequence $\frac{|m[x, T]|}{T^{\beta}}$ converges in distribution (cf. [25, p. 188]). Denote the limit distribution by $\nu$ (seen as a probability measure on the real line). Then

$$
\mu\left(\frac{|m[x, T]|}{T^{\beta}} \geq(a / \varepsilon)^{1 / \alpha}\right) \rightarrow \nu\left(|t| \geq(a / \varepsilon)^{1 / \alpha}\right)
$$


and hence there exists $T_{\varepsilon} \geq T_{0}$ such that

$$
\mu\left(\left|W_{T}\right| \geq a\right) \leq \nu\left(|t| \geq(a / \varepsilon)^{1 / \alpha}\right)+3 \varepsilon
$$

for $T \geq T_{\varepsilon}$. Since $\nu(|t| \geq A) \rightarrow 0$ as $A \rightarrow \infty$, this completes the proof.

Proof of Theorem 1.3 Hypotheses (i) and (ii) of the theorem translate immediately into the corresponding hypotheses for Lemma 3.4. Hence $W_{T} \rightarrow_{d} 0$ on $X$. By Lemmas 3.1 and $3.3, \frac{1}{\sqrt{T}} \widehat{\Phi}_{n[x, T]} \rightarrow_{d} G / \sqrt{\bar{r}}$ on $X_{r}$. Finally, by Lemma 2.1(b) with $p=2, \frac{1}{\sqrt{T}} \phi_{T} \rightarrow_{d} G / \sqrt{\bar{r}}$ on $X_{r}$.

\section{LIL and ASIP for suspension flows}

In this section, we show how to extend the LIL and ASIP from observations on $X$ to observations on the suspension flow $X_{r}$. As usual, $\phi: X_{r} \rightarrow \mathbb{R}$ has mean zero and $\Phi(x)=\int_{0}^{r(x)} \phi(x, u) d u$.

We say that $\phi$ satisfies the LIL if

$$
\limsup _{T \rightarrow \infty} \frac{\phi_{T}}{\sqrt{2 T \log \log T}}=\sigma \text { a.e. }
$$

and similarly for $\Phi$.

Theorem 4.1 Suppose that $S: X \rightarrow X$ is a measure-preserving transformation and that $S_{t}: X_{r} \rightarrow X_{r}$ is the suspension flow corresponding to a roof function $r$ : $X \rightarrow \mathbb{R}$ satisfying the SLLN (2.1). Suppose that $r \in L^{a}(X)$ and $\phi \in L^{b}\left(X_{r}\right)$ where $\left(1-\frac{1}{a}\right)\left(1-\frac{1}{b}\right) \geq \frac{1}{2}$.

If $\Phi$ satisfies the LIL with variance $\sigma_{1}^{2}$, then $\phi$ satisfies the LIL with variance $\sigma^{2}=\sigma_{1}^{2} / \bar{r}$

Proof Define $q:(3, \infty) \rightarrow(0, \infty)$ by $q(T)=(2 T \log \log T)^{-1 / 2}$. Noting that $T \mapsto$ $n[x, T]$ is a monotone surjection, we deduce that $\lim \sup _{T \rightarrow \infty} q(n[x, T]) \Phi_{n[x, T]}(x)=\sigma_{1}$ a.e. By (2.3), $\limsup _{T \rightarrow \infty} q(T) \Phi_{n[x, T]}(x)=\sigma$ a.e. It follows from Lemma 2.1(a) that

$$
\limsup _{T \rightarrow \infty} q(T) \phi_{T}(x, 0)=\sigma \text { a.e. }
$$

Choose $X^{\prime} \subset X$ with $\mu\left(X^{\prime}\right)=1$ such that (4.1) holds for all $x \in X^{\prime}$. Then

$$
\limsup _{T \rightarrow \infty} q(T) \phi_{T}(x, u)=\limsup _{T \rightarrow \infty} q(T) \phi_{T+u}(x, 0)=\limsup _{T \rightarrow \infty} q(T) \phi_{T}(x, 0)=\sigma
$$

for all $x \in X^{\prime}$ and $u \in[0, r(x)]$. 
We say that $\phi$ satisfies the ASIP if (after redefining $\left\{\phi_{T} \mid T \geq 0\right\}$ on a richer probability space without changing the joint distributions of $\left.\phi_{T}\right)$ there exists $\varepsilon>0$ and a Brownian motion $W$ with variance $\sigma^{2}$ such that $\phi_{T}=W(T)+o\left(T^{1 / 2-\varepsilon}\right)$ as $T \rightarrow \infty$ almost everywhere. Similarly for $\Phi$. Many statistical laws including the CLT and LIL are implied by the ASIP, see Philipp and Stout [24].

Theorem 4.2 Let $S_{t}: X_{r} \rightarrow X_{r}$ be the suspension flow corresponding to a roof function $r: X \rightarrow \mathbb{R}$. Suppose that $r \in L^{a}(X)$ and $\phi \in L^{b}\left(X_{r}\right)$ where $\left(1-\frac{1}{a}\right)\left(1-\frac{1}{b}\right)>\frac{1}{2}$. Suppose further that

$$
r_{N}=N \bar{r}+o\left(N^{1-\delta}\right) \text { as } N \rightarrow \infty
$$

almost everywhere, for some $\delta>0$.

If $\Phi$ satisfies the ASIP with variance $\sigma_{1}^{2}$, then $\phi$ satisfies the ASIP with variance $\sigma^{2}=\sigma_{1}^{2} / \bar{r}$.

Proof By assumption (shrinking $\delta$ if necessary), there is a sequence of random variables $\left\{S_{N}, N \geq 1\right\}$ equal in distribution to the sequence $\left\{\Phi_{N}, N \geq 1\right\}$ such that

$$
S_{N}=W_{1}(N)+o\left(N^{1 / 2-\delta}\right) \text { as } N \rightarrow \infty
$$

almost everywhere, where $W_{1}$ is a Brownian motion with variance $\sigma_{1}^{2}$.

Thanks to condition (4.2), we can strengthen (2.3) to conclude that

$$
n[x, T]=T / \bar{r}+o\left(T^{1-2 \delta}\right) \text { as } T \rightarrow \infty
$$

almost everywhere, where for convenience we have replaced $\delta$ by $2 \delta$.

Shrinking $\delta$ once again as necessary so that $\left(1-\frac{1}{a}\right)\left(1-\frac{1}{b}\right)>\frac{1}{2}+\delta$, Lemma 2.1(a) guarantees that

$$
\phi_{T}(x, 0)=\Phi_{n[x, T]}(x)+o\left(T^{1 / 2-\delta}\right) \text { as } T \rightarrow \infty \text { a.e. }
$$

Moreover, $r$ is finite almost everywhere, so for $\mu$-a.e. $x$ and each $u \in[0, r(x)]$,

$$
\phi_{T}(x, u)=\phi_{T+u}(x, 0)=\Phi_{n[x, T+u]}(x)+o\left(T^{1 / 2-\delta}\right) \text { as } T \rightarrow \infty .
$$

As in $\left[24\right.$, p. 23], without loss we may identify $\Phi_{N}$ with $S_{N}$ and we may suppose that they are defined on the same probability space as $\phi_{T}$ and $W_{1}$. Hence combining (4.3) and (4.5) yields

$$
\phi_{T}(x, u)=W_{1}(n[x, T+u])+o\left(T^{1 / 2-\delta}\right) \text { as } T \rightarrow \infty \text { a.e. }
$$


By equation (4.4), $n[x, T+u]=T / \bar{r}+o\left(T^{1-2 \delta}\right)$ and so it follows as in $[24$, p. 10 and p. 24] that for any $\delta^{\prime}<\delta$,

$$
W_{1}(n[x, T+u])=W_{1}(T / \bar{r})+o\left(T^{1 / 2-\delta^{\prime}}\right)=W(T)+o\left(T^{1 / 2-\delta^{\prime}}\right),
$$

where $W$ is a Brownian motion with variance $\sigma^{2}=\sigma_{1}^{2} / \bar{r}$. Hence

$$
\phi_{T}(x, u)=W(T)+o\left(T^{1 / 2-\delta^{\prime}}\right) \text { as } T \rightarrow \infty
$$

almost everywhere, as required.

Remark 4.3 (a) Condition (4.2) is satisfied if $r \in L^{2}(X)$ and $r$ is cohomologous in $L^{2}$ to a martingale [13, p. 238]. A result of Gordin [16] guarantees that $r$ is cohomologous to a martingale in a wide class of examples.

(b) Improved error terms in the ASIP for $\Phi$ and in (4.2) can be used to obtain an improved error term in the ASIP for $\phi$. So if $\Phi$ satisfies the ASIP with error $o\left(N^{1 / p}\right)$ for some $p>2$, then $\phi$ satisfies the ASIP with error $o\left(T^{1 / p^{\prime}}\right)$, for any $p^{\prime}<p$, provided that $r_{N}=N \bar{r}+o\left(N^{2 / p}\right)$ and $\left(1-\frac{1}{a}\right)\left(1-\frac{1}{b}\right) \geq\left(1-\frac{1}{p}\right)$.

(c) When $X$ is Axiom A, Hölder observations satisfy the ASIP with error term $o\left(N^{1 / 4+\alpha}\right)$ for any $\alpha>0$, see [14]. Moreover, Hölder roof functions satisfy the condition in part (a) (cf. $[9,14])$. Hence, when $r$ and $\phi$ are Hölder, we obtain the ASIP with error term $o\left(T^{1 / 4+\alpha}\right)$ improving slightly upon the result of [9].

\section{Applications}

Hyperbolic flows As discussed in the introduction, Ratner [25] established the CLT for Hölder observations on a hyperbolic basic set $X$, and also (by a quite complicated argument) the CLT for Hölder observations on the suspension $X_{r}$ by a Hölder roof function $r$. The CLT for general hyperbolic flows then follows from Bowen [3]. In this paper (see Theorem 1.1), we have given an elementary and general argument that yields the CLT for hyperbolic flows, based on the CLT for hyperbolic basic sets. (See Remark 1.2 for a comparison of the two proofs.)

Nondegeneracy The statistical limit theorems in this paper are said to be degenerate if $\sigma^{2}=0$ (equivalently $\sigma_{1}^{2}=0$ ). In our abstract setting, it is not possible to formulate useful criteria for nondegeneracy.

However, if $\Phi$ is a Hölder continuous observation on a hyperbolic basic set $X$ equipped with a Gibbs measure $\mu$, then it is well-known that $\sigma_{1}^{2}=0$ if and only if there is a constant $K_{1}$ such that $\left|\Phi_{N}\right|_{\infty} \leq K_{1}$ for all $N$, which is equivalent to the 
fact that $\Phi$ is a Hölder coboundary (see $[14, \S 5]$ for an explicit statement). Provided $r$ and $\phi$ are Hölder, so in particular $\phi_{T}(x, u)=\Phi_{n[x, T]}(x)+O(1)$, we conclude that $\sigma^{2}=0$ if and only if there is a constant $K$ such that $\left|\phi_{T}\right|_{\infty} \leq K$ for all $T$.

This condition for degeneracy has significant advantages over the $L^{2}$ condition in [25]. For continuous observations, it means that $\phi_{p}(x, u)=0$ whenever $S_{p}(x, u)=(x, u)$ (equivalently, $\Phi_{P}(x)=0$ whenever $S^{P} x=x$ ) so we can guarantee nondegeneracy by perturbing the observation along a single periodic trajectory.

Nonuniformly hyperbolic flows The CLT has been established for certain classes of nonuniformly hyperbolic and partially hyperbolic diffeomorphisms. The CLT for the suspension flow follows from Theorem 1.1.

For example, Young [30, 31] proves the CLT for smooth enough observations of a class of nonuniformly hyperbolic diffeomorphisms. By Theorem 1.1, we have

Corollary 5.1 Provided $r$ and $\phi$ are smooth enough, the CLT (and its functional version) hold for suspension flows over the nonuniformly hyperbolic transformations considered in [30, 31].

Nonuniformly hyperbolic diffeomorphisms The methods in this paper apply also to nonuniformly hyperbolic diffeomorphisms (and more generally to induced transformations). The tower construction in Young [30, 31] realises such diffeomorphisms as discrete suspensions over a uniformly hyperbolic base, where the roof function $r$ takes only integer values. Provided $r$ lies in $L^{2}$, the results in this paper immediately yield the CLT. The condition that $r$ is $L^{2}$ is more natural and general than the condition in [31] (though the emphasis there is on establishing rates of decay of correlations which is a much harder problem).

The application of the ideas in this paper to nonuniformly hyperbolic diffeomorphisms has been carried out recently by Gouëzel [18] (our main results are summarized in [18, Appendix A]). This provides a significantly simplified proof of results in Gouëzel [17].

A class of partially hyperbolic flows Suppose that $\Lambda$ is a hyperbolic basic set for a smooth flow $S_{t}$ with an equilibrium measure $\mu_{\Lambda}$ corresponding to a Hölder potential, and $G$ is a compact connected Lie group with Haar measure $\nu$. If $h_{t}: \Lambda \rightarrow G$ is a smooth cocycle $\left(h_{s+t}=h_{s} h_{t} \circ S_{s}\right)$, then we form the $G$-extension flow $S_{t, h}$ on $\Lambda \times G, S_{t, h}(y, g)=\left(S_{t}(y), g h_{t}(y)\right)$, with invariant measure $\mu_{\Lambda} \times \nu$. Such $G$-extensions are amongst the simplest examples of partially hyperbolic flows (We note that the arguments in $[9,25]$ do not seem to apply to such flows.) 
Theorem 5.2 Let $G$ be a semisimple compact connected Lie group and suppose that $\Lambda \times G$ is an ergodic $G$-extension of a hyperbolic basic set $\Lambda$ for a hyperbolic flow. Then the CLT (and its functional version) hold for sufficiently smooth observations $\phi: \Lambda \times G \rightarrow \mathbb{R}$. Moreover, the LIL and ASIP hold for sufficiently smooth observations.

Proof By Bowen [3], $\Lambda$ can be modelled as a symbolic flow $X_{r}$ where $X$ is a subshift of finite type, $r: X \rightarrow \mathbb{R}$ is a Hölder roof function, with equilibrium measure $\mu_{r}$ where $\mu$ is an equilibrium measure on $X$. Hence $\Lambda \times G$ is realised as $X_{r} \times G \cong(X \times G)_{r}$ with ergodic measure $(\mu \times \nu)_{r}$. (We regard $r$ as defined on $X \times G$ but independent of the $G$-component.) Since $\Lambda \times G$ is ergodic, it follows that $\mu \times \nu$ is an ergodic measure on $X \times G$.

Dolgopyat [10, Corollary 4.8] proves rapid mixing for Hölder observations on $X \times G$ that are sufficiently smooth in the $G$ direction and deduces the CLT using [20]. The CLT follows also from [22] and so does the ASIP. By the results in this paper, these results lift to the suspension $(X \times G)_{r}$ and hence to $\Lambda \times G$.

Remark 5.3 (Application to frame flows) Let $V$ be a negatively-curved orientable $n$-dimensional manifold, with unit tangent bundle $M=S V$. Let $\widehat{M}$ be the space of positively oriented orthonormal $n$-frames in $T V$. The frame flow on $\widehat{M}$ is an $\mathbf{S O}(n-1)$-extension of the geodesic flow on $M$. Recall that $\mathbf{S O}(n-1)$ is semisimple provided $n \geq 4$. Ergodicity of the frame flow has been proven by [4] when $n$ is odd, $n \neq 7$, and by $[5,6]$ in the remaining cases under a pinching condition on the curvature. By Theorem 5.2, the ASIP etc. hold for sufficiently smooth observations on such frame flows.

If $G$ is not assumed to be semisimple, then the situation is more complicated. Below, we describe some results for suspensions $(X \times G)_{r}$ where $X$ is a hyperbolic basic set for a diffeomorphism. Note that the hypotheses are now on $X \times G$ and for this reason the results are considerably weaker than in the semisimple case.

Suppose that $X$ is a hyperbolic basic set with Gibbs measure $\mu$ and $G$ is a compact connected Lie group with Haar measure $\nu$. If $S: X \rightarrow X$ is the underlying Axiom A dynamics and $h: X \rightarrow G$ is a smooth cocycle, the $G$-extension $S_{h}: X \times G \rightarrow X \times G$ is given by $S_{h}(x, g)=(S x, g h(x))$. Field et al. [15] showed that the $G$-extension $X \times G$ is ergodic with respect to $\mu \times \nu$ for an open dense set of smooth extensions $h: X \rightarrow G$. Such a $G$-extension $S_{h}$ is said to be stably ergodic.

For restricted classes of $G$-extensions ( $G$ semisimple covered above, but also $X$ Anosov infranilmanifold), Dolgopyat [10] proved that stable ergodicity implies rapid decay of correlations, and hence the CLT, for sufficiently regular observations $\Phi$ : $X \times G \rightarrow \mathbb{R}$. 
Corollary 5.4 Provided $r$ and $\phi$ are smooth enough, the CLT (and its functional version) hold for suspension flows over the G-extensions considered in [10].

In the context of equivariant flows, it is natural to consider equivariant observations $[23,14]$, and stronger results are possible.

Theorem 5.5 Let $G$ be a compact connected Lie group and suppose that $Y \times G$ is an ergodic G-extension of a hyperbolic basic set $Y$ for a hyperbolic flow. Let $\rho$ be a representation of $G$ on the vector space $\mathbb{R}^{k}$, and consider mean zero observations $\phi: Y \times G \rightarrow \mathbb{R}^{k}$ of the form $\phi(y, g)=\rho_{g} v(y)$, where $v: Y \rightarrow \mathbb{R}^{k}$ is Hölder. Then $\phi$ satisfies a $k$-dimensional $C L T$, and each one-dimensional projection of $\phi$ satisfies the ASIP.

Furthermore, the CLT is degenerate (singular covariance matrix) if and only if some component of $\phi_{T}$ is uniformly bounded, and the CLT is nondegenerate for an open and dense set of equivariant Hölder observations $\phi$.

Proof As usual, we can reduce to the case where $Y=X_{r}$ is the suspension of a subshift of finite type $X$ and $r$ is Hölder. Then $Y \times G=X_{r} \times G=(X \times G)_{r}$.

Define $\Phi(x, g)=\int_{0}^{r(x)} \phi(x, g, u) d u=\rho_{g} V(x)$ where $V(x)=\int_{0}^{r(x)} v(x, u) d u$. Then $\Phi: X \times G \rightarrow \mathbb{R}^{k}$ is a Hölder equivariant observation and it follows from $[14,21]$ that $\Phi$ satisfies the CLT. The CLT for $\phi$ follows from our main results and the Cramer-Wold technique. Similarly, it follows from [14] and Theorem 4.2 that each one-dimensional projection of $\phi$ satisfies the ASIP.

As in the Axiom A case, the statements about nondegeneracy for $\phi$ follow from the corresponding statements for $\Phi$ which were obtained by Nicol et al. [23].

Remark 5.6 The ideas in [14, 23] were applied by Ashwin et al. [2] to provide an explanation of hypermeander of spiral waves in planar excitable media. There, the spiral tip undergoes Brownian-like motion in the plane. Theorem 5.5 bridges the gap between discrete and continuous time [2, Section 5].

Acknowledgments This research was supported in part by NSF Grant DMS0244529, by the ESF "Probabilistic methods in non-hyperbolic dynamics" (PRODYN) programme, and by EPSRC GR/R87543/01. We are grateful to Francois Ledrappier and Roland Zweimüller for helpful discussions. We are also grateful to the referee for several useful suggestions and in particular for pointing out to us the application to frame flows in Theorem 5.2 and Remark 5.3. 


\section{References}

[1] J. Aaronson. An Introduction to Infinite Ergodic Theory. Amer. Math. Soc. 1997.

[2] P. Ashwin, I. Melbourne and M. Nicol. Hypermeander of spirals; local bifurcations and statistical properties. Physica D 156 (2001) 364-382.

[3] R. Bowen. Symbolic dynamics for hyperbolic flows. Amer. J. Math. 95 (1973) 429-460.

[4] M. Brin and M. Gromov. On the ergodicity of frame flows. Invent. Math. 60 (1980) 1-7.

[5] M. Brin and H. Karcher. Frame flows on manifolds with pinched negative curvature. Compositio Math. 52 (1984) 275-297.

[6] K. Burns and M. Pollicott. Stable ergodicity and frame flows. Geometriae Dedicata 98 (2003) 189-210.

[7] J.-P. Conze and S. Le Borgne. Méthode de martingales et flow géodésique sur une surface de courbure constante négative. Ergod. Th. E Dynam. Sys. 21 (2001), 421-441.

[8] I. P. Cornfeld, S. V. Fomin and Y. G. Sinai. Ergodic theory. Grundlehren der mathematischen Wissenschaften 245, Springer, New York, 1982.

[9] M. Denker and W. Philipp. Approximation by Brownian motion for Gibbs measures and flows under a function. Ergod. Th. E Dynam. Sys. 4 (1984) 541-552.

[10] D. Dolgopyat. On mixing properties of compact group extensions of hyperbolic systems. Israel J. Math. 130 (2002) 157-205.

[11] D. Dolgopyat. Limit theorems for partially hyperbolic systems. Trans. Amer. Math. Soc. 356 (2004) 1637-1689.

[12] G. K. Eagleson. Some simple conditions for limit theorems to be mixing. Teor. Verojatnost. i Primenen. 21 (1976) 653-660. (English translation: Theor. Prob. Appl. 21 (1976) 637-642, 1977.)

[13] W. Feller. An Introduction to Probability Theory and its Applications, II. Wiley, New York, 1966. 
[14] M. J. Field, I. Melbourne and A. Török. Decay of correlations, central limit theorems and approximation by Brownian motion for compact Lie group extensions. Ergod. Th. \&3 Dynam. Sys. 23 (2003) 87-110.

[15] M. J. Field, I. Melbourne and A. Török. Stable ergodicity for smooth compact Lie group extensions of hyperbolic basic sets. Ergod. Th. \& Dynam. Sys. To appear.

[16] M. I. Gordin. The central limit theorem for stationary processes. Soviet Math. Dokl. 10 (1969) 1174-1176.

[17] S. Gouëzel. Central limit theorem and stable laws for intermittent maps. Prob. Theory and Rel. Fields 128 (2004) 82-122.

[18] S. Gouëzel. Statistical properties of a skew product with a curve of neutral points. Preprint, 2004.

[19] F. Hofbauer and G. Keller. Ergodic properties of invariant measures for piecewise monotonic transformations. Math. Z. 180 (1982) 119-140.

[20] C. Liverani. Central limit theorem for deterministic systems. In: International Conference on Dynamical Systems (F. Ledrappier, J. Lewowicz, and S. Newhouse, eds.), Pitman Research Notes in Math. 362, Longman Group Ltd, Harlow, 1996, pp. 56-75.

[21] I. Melbourne and M. Nicol. Statistical properties of endomorphisms and compact group extensions. J. London Math. Soc. To appear.

[22] I. Melbourne and A. Török. Central limit theorems and invariance principles for time-one maps of hyperbolic flows. Commun. Math. Physics 229 (2002) 57-71.

[23] M. Nicol, I. Melbourne and P. Ashwin. Euclidean extensions of dynamical systems. Nonlinearity 14 (2001) 275-300.

[24] W. Philipp and W. F. Stout. Almost Sure Invariance Principles for Partial Sums of Weakly Dependent Random Variables. Memoirs of the Amer. Math. Soc. 161, Amer. Math. Soc., Providence, RI, 1975.

[25] M. Ratner. The central limit theorem for geodesic flows on $n$-dimensional manifolds of negative curvature. Israel J. Math. 16 (1973) 181-197.

[26] A. Rényi. On mixing sequences of sets. Acta Math. Acad. Sci. Hungar. 9 (1958) 215-228. 
[27] D. Ruelle. Thermodynamic Formalism. Encyclopedia of Math. and its Applications 5, Addison Wesley, Massachusetts, 1978.

[28] D. Ruelle. The thermodynamic formalism for expanding maps. Commun. Math. Phys. 125 (1989) 239-262.

[29] S. Wong. Law of the iterated logarithm for transitive $C^{2}$ Anosov flows and semiflows over maps of the interval. Monatsh. Math. 94 (1982) 163-173.

[30] L.-S. Young. Statistical properties of dynamical systems with some hyperbolicity. Ann. of Math. 147 (1998) 585-650.

[31] L.-S. Young. Recurrence times and rates of mixing. Israel J. Math. 110 (1999) $153-188$. 